\title{
Association of rates of household handgun ownership, lifetime major depression, and serious suicidal thoughts with rates of suicide across US census regions
}

\author{
D Hemenway, M Miller
}

See end of article for authors' affiliations

.....................

Correspondence to: Dr David Hemenway, Harvard School of Public Health, 677 Huntington Avenue, Boston, MA 02115, USA

hemenway@

hsph.harvard.edu
Injury Prevention 2002;8:313-316

Objective: Cross sectional studies in the United States often find a significant positive association between levels of household firearm ownership and suicide rates. This study investigates whether the association can be explained by differences in levels of mental health.

Methods: The relationship between household handgun ownership and overall suicide rates across United States regions after accounting for two mental health variables-lifetime prevalence of major depression and serious suicidal thoughts - were examined. Analyses also add another control variable (urbanization, education, unemployment, or alcohol consumption). Data on mental health variables come from the National Comorbidity Study, conducted in the early 1990s. Data on household handgun ownership come from the General Social Surveys.

Results: Across the nine regions for the early 1990s $(n=9)$, household handgun ownership rates are positively correlated with the suicide rate $(r=0.59)$ and are not correlated with either the lifetime prevalence of major depression or suicidal thoughts. After controlling for major depression and suicidal thoughts (and any of the four additional control variables), handgun ownership rates remain significantly associated with the overall suicide rate.

Conclusions: In United States regions with higher levels of household handgun ownership, there are higher suicide rates. This relationship cannot be explained by differences in the prevalence of two mental health indicators - lifetime rates of either major depression or suicidal thoughts.

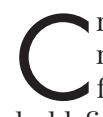
ross sectional studies in the United States, across regions, ${ }^{12}$ states, ${ }^{3-5}$ and urban counties or cities, ${ }^{67}$ often find a significant association between rates of household firearm ownership and suicide rates. ${ }^{8}$ Control variables have included urbanicity, education, unemployment and alcohol problems, but no ecological study we know of has controlled for possible mental health confounders.

In this article we use data from the National Comorbidity Survey (NCS) to investigate the regional relationship of handgun ownership levels and two psychological measureslifetime prevalence of major depression and serious suicidal thoughts-with rates of suicide.

Our primary aim is to begin to investigate whether the association between firearm prevalence and suicide can be explained by differences in levels of mental health.

\section{METHODS}

This study focuses on three related dependent variables: the total suicide rate, the firearm suicide rate, and the non-firearm suicide rate across the nine census regions. We begin with three explanatory variables, which are included in all analyses: rates of household handgun ownership, lifetime prevalence of major depression, and lifetime prevalence of serious suicidal thoughts. We also add a fourth explanatory variable-which is $(1)$ the percent of the population living in metropolitan areas, or (2) the percent with at least some college, or (3) the percent unemployed, or (4) the per capita alcohol consumption. These four control variables are added, one at a time, to regressions that include (as independent variables) the prevalence of household handgun ownership, lifetime suicidal thoughts, and lifetime major depression (that is, these regressions have four rather than three independent variables).

For suicides, we use average regional rates for a 10 year period (1988-97). Data for the number of suicides, firearm suicides, and non-firearm suicides as well as yearly population figures come from the National Center for Health Statistics Mortality Files. ${ }^{9}$ Deaths from firearm of undetermined intention constitute less than $3 \%$ of all firearm deaths and are excluded from the analysis. Region specific population and mortality data are derived by aggregating the corresponding state based data. The suicide rate within each region is very stable over the 10 year study period; the largest absolute (and percentage) variation in the rate of suicide within a region occurred in region 3, where the suicide rate averaged over the 10 year period was 10.9 per 100000 (range 9.9-11.6 per $100000)$. This within-region variation is far smaller than the twofold variation across regions. Using the rate for any year, or the average over the 10 years, yields similar results.

Data for the percentage of households with handguns in each region come from published survey based estimates from the General Social Surveys (GSS). The GSS, conducted by the National Opinion Research Center most years from 1972 to 1993, and biennially since 1994, is the gold standard for national surveys of gun ownership. In its current form, the GSS is conducted in person with a national area probability sample of 3000 non-institutionalized adults. The sample is chosen to be representative of each of the nine census regions. We use regional handgun ownership data averaged over the 1988-97 period. $^{10}$

Because the stock of guns in the United States is so high (over 200 million guns in civilian hands) and because guns are highly durable goods, year-to-year variations in survey estimates of firearm ownership rates are as likely to reflect measurement error rather than actual fluctuations in firearm

Abbreviations: GSS, General Social Surveys; NCS, National Comorbidity Survey 
Table 1 Regional rates of suicide, mental illness, and handgun ownership

\begin{tabular}{|c|c|c|c|c|c|c|c|c|c|}
\hline & $\begin{array}{l}\text { Region } 1 \\
\text { (NE) }\end{array}$ & $\begin{array}{l}\text { Region } 2 \\
\text { (MA) }\end{array}$ & $\begin{array}{l}\text { Region } 3 \\
\text { (ENC) }\end{array}$ & $\begin{array}{l}\text { Region } 4 \\
\text { (WNC) }\end{array}$ & $\begin{array}{l}\text { Region } 5 \\
\text { (SA) }\end{array}$ & $\begin{array}{l}\text { Region } 6 \\
\text { (ESC) }\end{array}$ & $\begin{array}{l}\text { Region } 7 \\
\text { (WSC) }\end{array}$ & $\begin{array}{l}\text { Region } 8 \\
\text { (MT) }\end{array}$ & $\begin{array}{l}\text { Region } 9 \\
\text { (PAC) }\end{array}$ \\
\hline Suicide rate (per 100 000) & 9.7 & 9.0 & 10.9 & 12.2 & 13.1 & 12.9 & 12.7 & 18.0 & 12.6 \\
\hline Firearm suicide rate (per 100 000) & 4.2 & 4.0 & 6.1 & 7.2 & 8.6 & 9.8 & 9.0 & 11.6 & 6.8 \\
\hline Non-firearm suicide rate (per 100 000) & 5.5 & 5.0 & 4.8 & 4.9 & 4.5 & 3.1 & 3.8 & 6.4 & 5.8 \\
\hline Major depression (prevalence, \%) & 18.5 & 15.3 & 17.1 & 16.4 & 16.5 & 16.8 & 15.2 & 16.7 & 20.9 \\
\hline Suicidal thoughts (prevalence, \%) & 14.5 & 11.9 & 14.2 & 12.7 & 13.0 & 12.7 & 11.0 & 19.9 & 15.2 \\
\hline Household handgun (prevalence, \%) & 14.1 & 11.9 & 19.5 & 19.2 & 27.3 & 38.3 & 30.7 & 26.4 & 22.5 \\
\hline
\end{tabular}

ENC, East North Central; ESC, East South Central; MA, Mid-Atlantic; MT, Mountain; NE, Northeast; PAC, Pacific; SA, South Atlantic; WNC, West North Central; WSC, West South Central.

ownership levels. Handgun ownership data are, therefore, averaged over the 10 year study period to obtain more reliable estimates of regional handgun ownership rates.

Data on mental health variables come from the NCS, a nationally representative household survey of people aged 15-54 years old. The NCS was conducted between September 1990 and February 1992 to determine the prevalence, distribution, correlates, and consequences of psychiatric disorders in the United States. The final sample consisted of 8098 participants for a response rate of over $82 \%$. The survey provides representative samples of the nine census regions. Verbal informed consent was obtained from all respondents and from the parents of minors before the interviews began. Detailed descriptions of the methods employed by the NCS have been published elsewhere. ${ }^{11}$

Mental health variables come from a survey of 15-54 year olds, whereas the suicide data pertain to all ages. Since rates of depression and suicide may be higher in more elderly groups, analyses might be biased if regional age distributions materially differ. However, across the nine regions, the percent of the population that is $15-54$ years old varies little: from $70 \%$ to $73 \%$ with a mean of $72 \%$. Moreover, the correlation coefficient across the nine regions between the suicide rate for all individuals and for individuals $15-54$ years old is 0.99 . Not surprisingly, results are virtually identical whether the dependent variable is the regional suicide rate for all individuals or the regional suicide rates for individuals aged 15-54 year olds.

The NCS provides information concerning the prevalence of a multitude of psychiatric disorders, including mood disorders (depression, dysthymia, mania), anxiety disorders (agoraphobia, generalized anxiety disorder, panic, social phobia, simple phobia, post-traumatic stress disorders), and substance use disorders (alcohol abuse, alcohol dependence, drug abuse, drug dependence).

All respondents who screened positive for a lifetime prevalence of any disorder and a random subsample of other respondents were administered a part II interview (5877 respondents). The data for these respondents were weighted to correct for differential probabilities of selection into part II as well as for differential probabilities of within-household selection and non-response. Questions on three aspects of suicidality were included in the part II interview (suicidal thoughts, plans, attempts).

Since the number of observations in our study is very small $(\mathrm{n}=9$ regions $)$, we decided to investigate only a small number of disorders. The criteria for selection were a strong expected relationship between the disorder and suicide based on the psychological literature, and a large enough prevalence of the disorder in the population so the attributable risk would be sufficient to discover an effect and there would be an adequate cell size to ensure precision of the regional estimates. For the second criteria, we decided that the weighted national prevalence of the disorder had to be great enough to ensure that, if the least populous region had an average prevalence, there would be a minimum of 40 respondents from that region reporting the disorder. Two disorders met our criteria: lifetime prevalence of major depression and of serious suicidal thoughts.

The question on suicide thoughts asked: "Have you ever seriously thought about committing suicide?" The prevalence, distribution, and risk factors for lifetime prevalence of ever having serious suicidal thoughts is described in detail elsewhere. ${ }^{12}$

Major depression is the prevalence of people having a lifetime major depressive episode, based on responses to a modified version of the Composite International Diagnostic Interview. The approach to case ascertainment in the NCS, and particularly the comparison with the use of the Diagnostic Interview Schedule in the Epidemiologic Catchment Area study is described in detail elsewhere. ${ }^{13}$

Data for the percent of the population living in metropolitan areas, the percent with at least some college, and the percent unemployed are 10 year averages and come from the Statistical Abstract of the United States. Data for alcohol consumption pertains to gallons of beer, wine, or spirits divided by the population over age 14 and come from the National Institute on Alcohol Abuse and Alcoholism.

Bivariate associations are presented as simple correlation coefficients. Multivariate models use ordinary least squares, using two tailed $t$ tests for statistical significance.

\section{RESULTS}

Across the nine regions, the suicide rate, averaged from 198897, varies from 9.0 per 100000 in the Mid-Atlantic region to 18.0 in the Mountain region. Table 1 provides the regional rates of suicide, firearm suicide and non-firearm suicide, the lifetime prevalence of major depression and suicidal thoughts, and the average rates of household handgun ownership over the 10 year period. Seventeen percent ( $17 \%$ ) of respondents on the NCS study reported ever having a major depression; residents of the Pacific region had the highest prevalence. Over $13 \%$ of respondents reported ever having serious suicidal thoughts; the Mountain region had the highest prevalence. Rates of household handgun ownership range from $12 \%$ in the Mid-Atlantic region to 38\% in the East South Central region.

Across the nine regions, household handgun ownership rates are highly positively correlated with the suicide rate ( $r=$ $0.59)$, and the firearm suicide rate $(r=0.83)$, and negatively correlated with the non-firearm suicide rate $(r=-0.57)$ (table 2 ). Household handgun ownership rates are not correlated with either the prevalence of major depression $(r=-0.10)$ or suicidal thoughts $(r=-0.01)$.

The prevalence of major depression and prevalence of suicidal thoughts are positively correlated, but the prevalence of major depression is not correlated with the suicide rate $(r=0.00)$, while the prevalence of suicidal thoughts is correlated with the suicide rate $(r=0.68)$ (table 2$)$. Figure 1 provides a graphical representation of regional suicide rates with household handgun ownership, prevalence of major depression, and suicidal thoughts across the nine regions. 
Table 2 Correlation coefficients: rates of suicide, firearm suicide, non-firearm suicide, lifetime major depression, lifetime suicidal thoughts and firearm ownership, US census regions

\begin{tabular}{llllll}
\hline & Suicide rate & $\begin{array}{l}\text { Firearm } \\
\text { suicide rate }\end{array}$ & $\begin{array}{l}\text { Non-firearm } \\
\text { suicide rate }\end{array}$ & $\begin{array}{l}\text { Major } \\
\text { depression }\end{array}$ & $\begin{array}{l}\text { Suicidal } \\
\text { thoughts }\end{array}$ \\
\hline Firearm suicide rate & 0.93 & & & & \\
Non-firearm suicide rate & 0.27 & -0.12 & & & \\
Major depression & 0.00 & -0.18 & 0.43 & 0.39 & \\
Suicidal thoughts & 0.68 & 0.39 & 0.77 & -0.10 & -0.01 \\
Household handgun prevalence & 0.59 & 0.83 & -0.57 & -0.10 \\
\hline
\end{tabular}

Rates of suicide, firearm suicide, and non-firearm suicide come from the National Center for Health Statistics. Psychiatric disorder prevalence estimates by census region, 1991-92, come from the National Co-morbidity Study. Lifetime major depression $(n=8098)$ and suicidal thoughts (that is, ever seriously considered suicide) $(n=5877)$ were reported by $17 \%$ and $13 \%$ of respondents nationally. Mean household firearm and handgun ownership rates come from the General Social Surveys (1988-97).

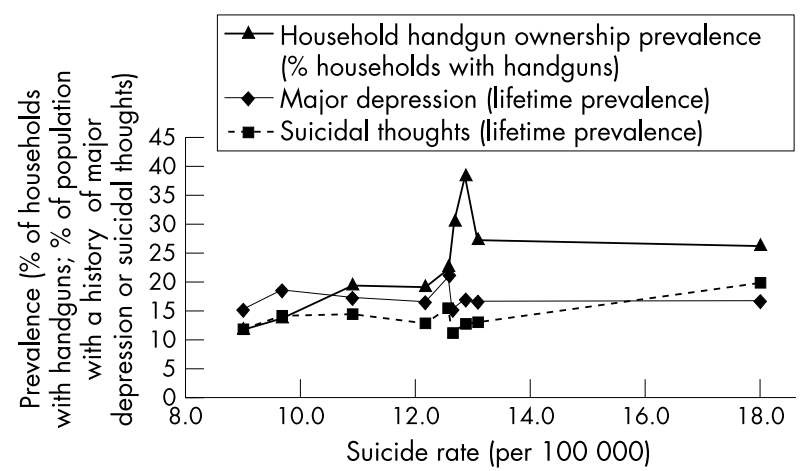

Figure 1 Regional rates of suicide compared with regional prevalence of household handgun ownership (1988-97), lifetime major depression, and lifetime suicidal thoughts.

In multivariate regression $(\mathrm{n}=9)$, with household handgun ownership, major depression and suicidal thoughts as the three independent variables, handgun ownership levels are positively associated with the firearm suicide rate $(\mathrm{p}<0.01)$, negatively associated with the non-firearm suicide rate $(\mathrm{p}<0.01)$, and positively associated with the overall suicide rate $(\mathrm{p}<0.05)$ (not shown). Suicidal thoughts are positively associated with all three suicide rates $(p<0.01)$, while major depression is not associated with any of the three suicide rates.

The Mountain region has the lowest population, and the highest rate of suicide. As a sensitivity analysis we eliminated that region from the regression $(\mathrm{n}=8)$. In multivariate analysis, the association of household handgun ownership levels with firearm, non-firearm and total suicide rates is unaffected, major depression remains non-significantly correlated with the suicide rates, but suicidal thoughts are no longer significantly correlated with any of the suicide rates (not shown). The simple correlation between suicidal thoughts and overall suicide falls dramatically $(r=-0.11)$, while the correlation between major depression and suicidal thoughts rises to $r=0.93$ (not shown).

When any one of the additional variables (the percent of the population living in metropolitan areas, the percent with at least some college, the percent unemployed, or the per capita alcohol consumption) are added as a fourth explanatory variable, the relationship between the regional prevalence of household handgun ownership and the regional suicide rate remains similar, statistically and in magnitude, as does the relationship between lifetime suicidal thoughts and the regional suicide rate (not shown).

\section{DISCUSSION}

Across United States regions, where there are more handguns there are higher rates of firearm suicide, lower rates of non-firearm suicide, but higher levels of overall suicide. This article shows that regional levels of handgun ownership are not correlated with regional lifetime rates of major depression or suicidal thoughts. The association between rates of handgun ownership and suicide remains strong at the regional level even after accounting for the lifetime prevalence of major depression and suicidal thoughts.

The association between household handgun ownership rates and the total suicide rate is robust. The handgun-suicide connection holds when we use the 1992 rates of suicide rather than the 1988-97 average (not shown) and when rates of gun ownership rather than just handgun ownership are used as an independent variable (though the association between gun ownership levels and suicide is not quite so strong) (not shown). In addition, our results hold when any one of four additional control variables are added to the analyses (urbanization, education, unemployment, or alcohol consumption).

This study has various limitations. First, as in any ecological study, a concern is that the association found at the aggregate level does not exist at the individual level. For example, from our data, even if there is a regional level association between alcohol and suicide, we do not know if the individuals who are alcohol consumers are the ones more likely to commit suicide. However, from other studies we know that guns are the prime method of suicide in the United States, most people who use guns to commit suicide use family guns, and a gun in the home is a risk factor for firearm suicide. ${ }^{8}$ We thus have somewhat less reason to be concerned about the "ecological fallacy" with respect to the gun prevalence-suicide connection.

Second, there is uncertainty associated with our measures-particularly our explanatory variables that come from self report surveys-that may bias the estimated relationships. Third, regional groupings undoubtedly mask considerable variation in the prevalence of both handgun ownership levels and mental health problems. Because we have representative mental health data only at the regional level, we have only nine observations. Fourth, we use only two measures of mental health-lifetime prevalence of major depression and of serious suicidal thoughts. Other mental health and socioeconomic factors undoubtedly influence the suicide rate.

We did not find any association between lifetime prevalence of major depression and suicide. Using data on current (30 day) prevalence of major depression might provide a better measure of susceptibility to suicide than lifetime prevalence. The NCS found a prevalence of current depression of less than $5 \%$, putting into question the precision of estimates for the least populous of the nine census regions, if we use that measure. Dividing the country in four regions, the prevalence of current depression was highest in the South (where gun prevalence and suicide rates are highest) but second highest in the Northeast (where gun prevalence and suicide rates are lowest). ${ }^{13}$ 


\section{Key points}

Across US regions:

- Handgun ownership rates are associated with suicide rates.

- Handgun ownership rates are not associated with rates of lifetime major depression.

- Handgun ownership rates are not associated with rates of lifetime serious suicidal thoughts.

- Rates of depression and suicidal thoughts cannot account for the handgun-suicide connection.

Compared with males, females have higher rates of both current and lifetime depression, higher rates of suicidal thoughts and suicidal attempts, but lower rates of suicide. Unfortunately, the NCS is not large enough to allow us to confidently disaggregate regional mental health measures by gender or age to get precise estimates to include in our analysis.

The strengths of our ecological study are that we use a good measure of handgun ownership levels, and we are the first to control for mental health risk factors. Our main results are consistent with case-control studies that find that a handgun or other firearm in the home is a risk factor for suicide, even after accounting for various measures of mental health. ${ }^{14-18}$ We find that in regions with higher levels of household handgun ownership, there are higher rates of firearm suicide, lower rates of non-firearm suicide (suggesting substitution of methods), and higher rates of overall suicide. This positive association between handgun prevalence and overall suicide across regions cannot be explained by differences in levels of two indicators of mental health problems-lifetime prevalence of major depression or suicidal thoughts.

\section{ACKNOWLEDGEMENT}

This research was supported in part by the Joyce Foundation, Robert Wood Johnson Foundation, and the Open Society Institute. Thanks to Beth Molnar and Ron Kessler.

Authors' affiliations

D Hemenway, M Miller, Harvard School of Public Health, Boston

\section{REFERENCES}

1 Markush R, Bartolucci A. Firearms and suicide in the United States. Am J Public Health 1984;64:123-7.

2 Birckmayer J, Hemenway D. Suicide and gun prevalence: are youth disproportionately affected? Suicide Life Threat Behav 2001;31:303-10. 3 Lester D. Gun ownership and suicide in the United States. Psychol Med 1989;19:519-21.

4 Miller M, Azrael D, Hemenway D. Firearm availability and unintentional firearm deaths, suicide and homicide among 5-14 year olds. J Trauma 2002;52:267-75

5 Miller M, Azrael D, Hemenway D. Firearm availability and unintentional firearm deaths, suicide and homicide among women. J Urban Health 2002;79:26-38

6 Kleck G, Patterson EB. The impact of gun control and gun ownership levels on violence rates. Journal of Quantitative Criminology 1993:9:249-87.

7 Hellsten JJ. Motivation and opportunity: an ecological investigation of US urban suicide, 1970-1990. PhD thesis in social ecology. Irvine: University of California, 1995.

8 Miller M, Hemenway D. The relationship between firearms and suicide: a review of the literature. Aggression and Violent Behavior 1999;4:59-75

9 National Center for Injury Prevention and Control, Centers for Disease Control and Prevention. Injury mortality statistics. Available at: http://www.wonder.cdc.gov/mortsql.shtml.

10 Davis J, Smith T. General social surveys, 1972-1998 [machine-readable data file]. Principal Investigator, James A Davis; Director and Co-Principal Investigator, Tom W Smith; Chicago: National Opinion Research Center. The Roper Center for Public Opinion Research, University of Connecticut, 1998.

11 Kessler RC, McGonagle KA, Zhao S, et al. Lifetime and 12 month prevalence of DSM-III-R psychiatric disorders in the United States. Results from the National Comorbidity Survey. Arch Gen Psychiatry 1994;51:8-19.

12 Kessler RC, Borges G, Walters EE. Prevalence of and risk factors for lifetime suicide attempts in the National Comorbidity Survey. Arch Gen Psychiatry 1999;56:617-26

13 Blazer DG, Kessler RC, McGonagle KA, et al. The prevalence and distribution of major depression in a national community sample: the National Comorbidity Survey. Am J Psychiatry 1994;151:979-86.

14 Brent DA, Perper JA, Goldstein CE, et al. Risk factors for adolescent suicide. A comparison of adolescent suicide victims with suicidal inpatients. Arch Gen Psychiatry 1988;45:581-8.

15 Brent DA, Perper JA, Allman CJ, et al. The presence and accessibility of firearms in the homes of adolescent suicides: a case-control study. JAMA 1991;266:2989-95.

16 Brent DA, Perper JA, Moritz G, et al. Firearms and adolescent suicide: a community case-control study. Am J Dis Child 1993;147:1066-71.

17 Bukstein OG, Brent DA, Perper JA, et al. Risk factors for completed suicide among adolescents with a lifetime history of substance abuse: a case-control study. Acta Psychiatr Scand 1993;88:403-8.

18 Kellermann AL, Rivara FP, Somes G, et al. Suicide in the home in relation to gun ownership. N Engl J Med 1992;327:467-72. 